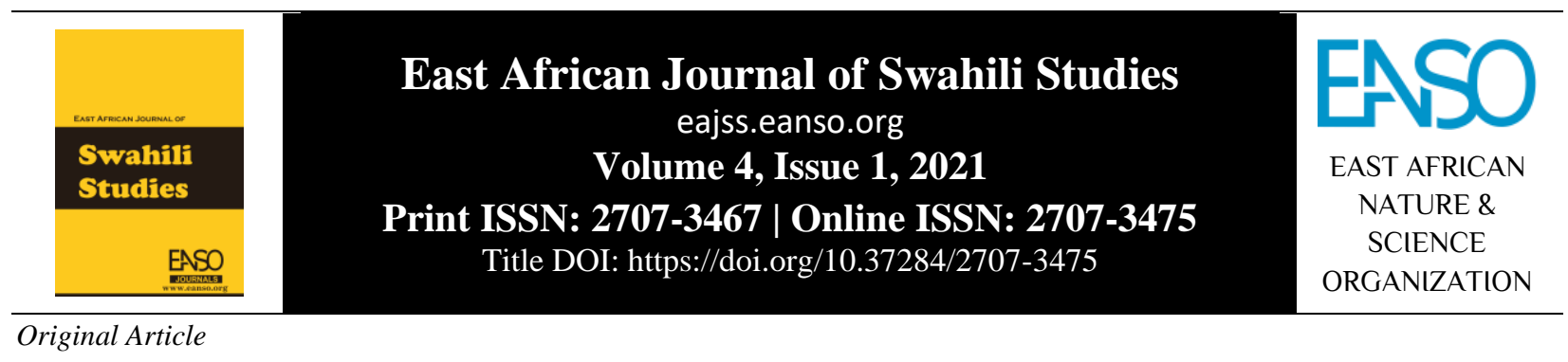

\title{
Uhusika katika Vichwa vya Habari za Siasa.
}

Fidelis Kioko Mutuku, ${ }^{*}$ Dkt. Jacktone Onyango, PhD ${ }^{1}$ na Dkt. Leonard Chacha Mwita, PhD

${ }^{1}$ Chuo Kikuu Cha Kenyatta, S.L.P. 248 - 90129, Ngwata, Kenya.

* Barua pepe ya mawasiliano: fmutuku413@gmail.com.

DOI ya Nakala: https://doi.org/10.37284/eajss.4.1.494

Tarehe ya Uchapishaji: $\quad$ IKISIRI

06 Desemba 2021 Uhusika ni dhana ya kisarufi ambayo hutumiwa kuonyesha dhima ya maneno katika tungo. Dhana hii ni muhimu katika uchambuzi wa tungo

Maneno Muhimu: kwani hunuia kuwasilisha ujumbe wa kisemantiki uliogubikwa katika muundo wa ndani. Makala hii inadhihirisha dhana mbalimbali za uhusika

Uhusika, na jinsi zinavyojitokeza katika vichwa vya habari za siasa zilizoko kwenye

Uhusika Kiima,

Uhusika Mahali,

Uhusika Yambwa,

Uhusika Tendea,

Uhusika Tokeo,

Uhusika Ala,

Muundo Ndani,

Muundo Nje. magazeti teule ya Taifa Leo. Nadharia ya uhusika iliyoasisiwa na Charles Fillmore mnamo mwaka wa 1968 imeongoza uchunguzi huu. Utafiti huu umefanywa baada ya kubaini kuwa wasomaji mbalimbali wa gazeti hufasiri maana anuwai kwenye kichwa kimoja cha habari za siasa. Jambo hili husababisha utata wa kimaana na uenezaji wa jumbe zisizo thabiti katika jamii, hivyo basi, kuzalisha migogoro ya kisiasa nchini. Upekuzi wa yaliyomo kwenye matoleo ya gazeti la Taifa Leo ambayo yametawaliwa na vichwa vya habari za siasa na kuchapishwa kati ya mwaka 2017-2019 ni msingi wa data ya utafiti huu. Vichwa vya habari za kisiasa kumi na nane vilisampulishwa kimakusudi na kutumika katika uchambuzi wa uchunguzi huu. Data katika makala hii imewasilishwa kupitia mfumo wa majedwali na maelezo. Matokeo ya utafiti huu yameshikilia kwamba, mahusiano kati ya kitenzi na nomino au kiwakilishi chake katika sentensi hubainisha aina ya uhusika. Uchunguzi huu una umuhimu kwa wasomi wa magazeti na taifa kijumla. Wasomaji wa magazeti wameongozwa na kigezo cha dhima ya maneno katika tungo ili kudondoa maana sahihi katika vichwa lengwa vya habari za kisiasa na kupitisha fasiri hiyo moja kwa wanajamii.

\section{APA CITATION}

Mutuku, F. K., Onyango, J., \& Mwita, L. C. (2021). Uhusika katika Vichwa vya Habari za Siasa. East African Journal of Swahili Studies, 4(1), 58-67. https://doi.org/10.37284/eajss.4.1.494

58 | This work is licensed under a Creative Commons Attribution 4.0 International License. 


\section{CHICAGO CITATION}

Mutuku, Fidelis Kioko, Jacktone Onyango, \& Leonard Chacha Mwita. 2021. "Uhusika katika Vichwa vya Habari za Siasa”. East African Journal of Swahili Studies 4(1), 58-67. https://doi.org/10.37284/eajss.4.1.494.

\section{HARVARD CITATION}

Mutuku, F. K., Onyango, J., \& Mwita, L. C. (2021) "Uhusika katika Vichwa vya Habari za Siasa", East African Journal of Swahili Studies, 4(1), pp. 58-67. doi: 10.37284/eajss.4.1.494.

\section{IEEE CITATION}

F. K. Mutuku, J. Onyango, \& L. C. Mwita, "Uhusika katika Vichwa vya Habari za Siasa”, EAJSS, vol. 4, no. 1, pp. 58-67, Dec. 2021.

\section{MLA CITATION}

Mutuku, Fidelis Kioko, Jacktone Onyango, \& Leonard Chacha Mwita. "Uhusika katika Vichwa vya Habari za Siasa". East African Journal of Swahili Studies, Vol. 4, no. 1, Dec 2021, pp. 58-67, doi:10.37284/eajss.4.1.494.

\section{UTANGULIZI}

Makala hii inadhihirisha dhana mbalimbali za uhusika na jinsi zinavyojitokeza katika vichwa vya habari za siasa zilizoko kwenye magazeti teule ya Taifa Leo. Katika kila dhana ya uhusika, vichwa vitatu vya habari vimeorodheshwa kama mifano ya sentensi zilizo na uhusika teule. Kichwa kimoja cha habari kati ya vitatu vilivyoorodheshwa kimechambuliwa kwa niaba ya vichwa vingine ndipo kuonyesha jinsi uhusika hujitokeza. Kwa mujibu wa Chomsky (1965), kila sentensi katika lugha huwa na kategoria mbili za uwakilisho, ambazo ni muundo ndani na muundo nje. Muundo ndani huwakilisha mahusiano msingi ya kisemantiki katika sentensi huku muundo nje ukiwakilisha umbo la kifonolojia la sentensi hiyo. Nadharia ya uhusika ya Fillmore (1968) ambayo imechambua vichwa vya habari teule imejkita mno kwenye kategoria ya uwakilisho wa muundo ndani wa sentensi.

Vichwa vilivyoko katika magazeti teule ya Taifa Leo vimewasilishwa kwa muundo wa nje. Fillmore (1968) amebainisha mfumo wa modeli ya uhusika na kanuni tatu kuu za utiaji uhusika katika sentensi. Mfumo huu wa modeli ya utiaji uhusika na kanuni hizi ndio uliotuongoza katika uundaji wa muundo ndani wa vichwa teule vya habari pamoja na utiaji uhusika faafu kwa vichwa hivyo.

\section{Tatizo Linaloshughulikiwa}

Utafiti huu umejikita katika uchunguzi wa jinsi uhusika ulivyojitokeza katika vichwa vya habari vya siasa vilivyoko kwenye gazeti la Taifa Leo. Kujitokeza kwa kipengele hiki cha sarufi cha uhusika kumefumbua fasili yenye usawa wa kimaana katika vichwa teule vya habari, kinyume na fasili nyingine binafsi ambazo hutegemea upana wa mawanda ya fikra za wasomi na uelewaji wao wenyewe. Fasili hizi za kibinafsi hutofautiana kutoka msomi mmoja hadi mwingine. Hivyo basi, maana mbalimbali za kichwa kimoja cha habari ya siasa huweza kuibuliwa na kusababisha utata wa kimaana katika ujumbe husika.

\section{Misingi ya Nadharia}

Utafiti huu umeongozwa na nadharia ya uhusika ambayo hujikita kwenye dhima ya maneno katika tungo. Nadharia hii iliasisiwa mwaka wa 1968 na mwanaisimu, Charles J. Fillmore (Fillmore 1968). Fillmore ni mwanaisimu wa Kimarekani aliyezaliwa mnamo mwaka wa 1929. Kwa mujibu wa Chafe (1970), Fillmore alikuwa mmoja kati ya wanaisimu ambao waliasisi uwakilisho wa utambuzi wa kiisimu ambao ulibainisha tofauti kati ya utambuzi wa lugha kisintaksia na kisemantiki. Mtazamo wa Fillmore (1968) kuhusu nadharia ya uhusika umejikita katika misingi miwili mikuu. Misingi hii ni; kitovu cha sintaksia na kategoria fiche.

Msingi wa kitovu cha sintaksia ni mtazamo ambao hutenda kazi kutoka umbo la mofolojia na maumbo mengine ya matawi ya isimu yaliyo chini ya umbo hili. Msingi huu hujikita katika matumizi maana ya kategoria za uhusika za kimapokeo. Kanuni za umbo la kisemantiki ni miundo ya ndani iliyoundwa na modeli ya uhusika ya 1968 ambayo hubainishwa kwa sifa zifuatazo;

- Sentensi hutenganishwa kwa kauli na hali.

S (Sentensi) $\rightarrow$ KL (Kauli) + HL (Hali) 
Kauli ni seti za mahusiano yanayohusisha vitenzi na nomino zisizokuwa na njeo ilhali hali huhusisha ukanushi, njeo na kadhalika.

- Kauli huhusisha kitenzi na mfuatano wa uhusika kutoka kulia hadi kushoto.

$\mathrm{KL}($ Kauli $) \rightarrow \mathrm{T}$ (Kitenzi) $+\mathrm{UH}+\mathrm{UH}_{\mathrm{n}} \ldots$ (Mfuatano wa uhusika)

Kauli huhusisha kitenzi na mfuatano wa ishara za uhusika za kirai nomino. Kitenzi huwekwa upande wa kushoto huku dhana za uhusika zilizo na mahusiano zikiorodheshwa kwa mpangilio kutoka sehemu ya kulia hadi kushoto pamoja na chaguo la kiima kilicho na uwezekano kwenye sehemu ya kulia katika muundo wa ndani.

- Ishara za uhusika hutokea katika muundo wa ndani.

$\mathrm{UH}($ Uhusika) $\rightarrow \mathrm{K}$ (Ishara ya uhusika) $+\mathrm{KN}$ (Kirai nomino)

Kila uhusika huhusisha ishara za uhusika na kirai nomino, ambapo ishara za uhusika hutanguliza kirai nomino katika muundo wa ndani. Ishara za uhusika ni kipengele bia cha lugha ambacho chafaa kutambulika kama kihusishi na kiambishi uhusika.

Katika uwasilishaji wa muundo msingi wa sentensi, kanuni hizi tatu hutumika kwa utaratibu rasmi. Dhana moja au mbalimbali za uhusika kama vile ala, mhusika, mtendi, yambwa na kadhalika hupishwa nafasi katika kategoria za uhusika za kanuni ya pili. Ishara za uhusika halisi ambazo huhusiana na uhusika hutiwa katika kanuni ya tatu. Kwa ujumla nadharia hii imejengeka katika mihimili mitatu mikuu ambayo ni; utambuzi wa muundo wa kimsingi wa sentensi ambao ni muhimu kwenye utambulishaji wa mahusiano ya kiuhusika, uwepo wa kanuni uhusika chujaji ambayo hutumika kuweka uhusika katika kila kirai nomino wazi cha lugha yoyote ile na uangaziaji wa mahusiano ya ndani ya kisemantiki.

\section{Mbinu za Ukusanyaji Data}

Kwa kuwa utafiti huu ni wa maktabani pekee, mtafiti ametumia mbinu ya upekuzi wa yaliyomo katika ukusanyaji wa data yake. Pia, mtafiti amesoma kwa kina magazeti teule ya Taifa Leo, yaliyochapishwa kati ya mwaka wa 2017 na 2019 kisha akabainisha sentensi zinazopatikana katika magazeti hayo kama vijianuani vya habari za siasa katika magazeti ya Taifa Leo. Jumla ya vichwa vya habari za kisiasa kumi na vinane vimeteuliwa kimakusudi kama data ya utafiti. Katika kila aina ya uhusika, vichwa vitatu vya habari za siasa vimetumika kama data teule. Kigezo cha sifa ya siasa katika vichwa vya habari teule kimeongoza uteuzi wa data lengwa. Kichwa kimoja cha habari kimechambuliwa kisintaksia katika kila aina ya uhusika na maelezo ya ziada kuambatanishwa na uchambuzi huo kama ilivyo kwenye sehemu inayofuata.

\section{Uhusika Kiima}

Uhusika kiima ni mojawapo ya aina sita za uhusika alizobainisha Fillmore (1968). Katika data teule ya utafiti, mtafiti ameambatisha mada tatu za kisiasa zenye muundo uhusika kiima kwa ajili ya uchambuzi. Kwa mujibu wa Fillmore (1968), nomino au kiwakilishi kinapotumika kama kiima cha kitenzi, hutambulishwa kuwa uhusika kiima. Huu ni uhusika ambao hujibu swali; ni nani aliyetenda kitendo fulani? Hivyo basi, uhusika huu huhusisha vitu vyenye uhai ambavyo husisimua utendaji wa tendo. Kando na kuhusisha viumbe hai pekee, roboti na taifa hujumuishwa kwenye uhusika kiima kwani ni vitu vyenye uwezo wa kutenda kitendo ambacho kiumbe hai chaweza kutenda.

Muundo wa baadhi ya vichwa vya habari za siasa kwenye gazeti la Taifa Leo ni wa uhusika kiima. Maneno yafuatayo: Musila, Uhuru na Wakazi hubainisha uwepo wa uhusika kiima katika vichwa teule vifuatavyo: Musila ajiondoa Wiper baada ya kushindwa katika mchujo, Mawaziri 13 watemwa na Uhuru na Wakazi wamtaka Rais nyumbani. Mchoroti ufuatao umekichambua kichwa cha habari; Wakazi wamtaka Rais nyumbani ndipo kuthibitisha jinsi mtenda wa kitendo huwa nguzo kuu katika utiaji uhusika kiima kwa sentensi. Lengo kuu la uchambuzi huu ni kujibu swali, ni nani aliyemtaka rais nyumbani?

\section{Kielelezo 1: Utiaji uhusika kiima}

- Muundo nje 


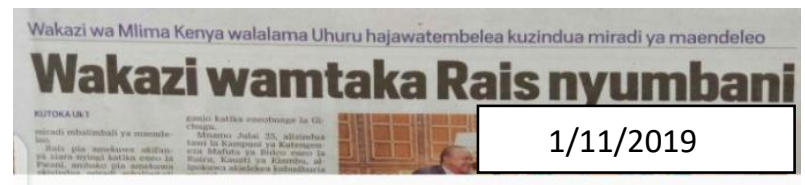

Kulingana na Massamba (2012), baada ya kuandika au kutamka maneno, umbo la nje la neno hilo hujitokeza. Muundo nje wa vichwa teule vya habari za siasa hujitokeza zisomwapo. Vichwa hivi vya habari huashiria jinsi vipashio mbalimbali vya lugha huungana na kuunda sentensi kamili na sahihi kisintaksia. Maana ibebwayo na sentensi hiyo hutokana na muundo wake wa ndani.

- Muundo ndani

Kwa mujibu wa Fillmore (1968), umbo la ndani la sentensi ni dhahania na ndilo kigezo kikuu cha maumbo na miundo yote ya lugha asilia ya

Mchoroti 1: Muundo wa uhusika kiima binadamu. Kwa hivyo, umbo la ndani hujengeka tu akilini na ndilo hubeba maana ya neno, hivyo ndilo maana.

Uchambuzi wa mchoroti ufuatao huonyesha jinsi kanuni tatu kuu za utiaji uhusika zake Fillmore (1968) hutenda kazi. Kanuni hizi hutenda kazi katika muundo ndani wa sentensi. Mtafiti anaonyesha wazi kuwa, kichwa hiki cha habari kimeundwa kwa sehemu mbili kuu, nazo ni: kauli na hali. Sehemu ya hali imehusisha kigezo cha wakati pekee; ambao ni wakati uliopo ilhali sehemu ya kauli imehusisha kitenzi kikuu na mfuatano wa dhana za uhusika mbalimbali kama vile; uhusika yambwa, uhusika mahali na uhusika kiima. Ndipo uhusika utiwe katika sentensi, ishara za uhusika hutanguliza kirai nomino kama inavyoonyeshwa katika mchoroti huu na kanuni ya tatu.

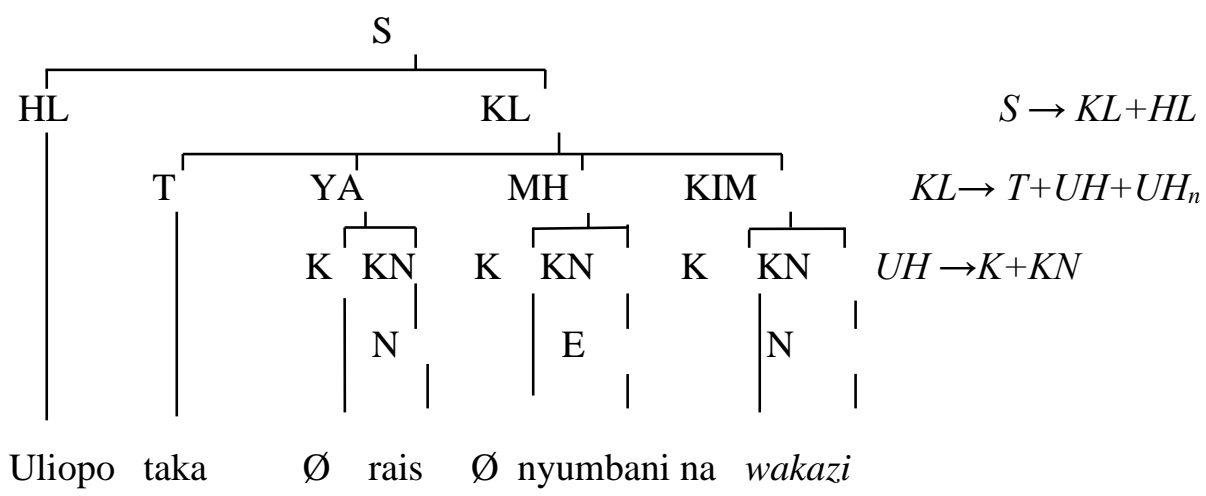

Tanbihi: S (Sentensi), KL (Kauli), HL (Hali), UH (Uhusika), $\mathrm{UH}_{\mathrm{n}}$ (Mfuatano wa uhusika), KIM (Uhusika kiima), MH (Uhusika mahali), YA (Uhusika Yambwa), K (Ishara ya uhusika), KN (Kirai nomino), $\mathrm{T}$ (Kitenzi), $\mathrm{N}$ (Nomino) na $\mathrm{E}$ (Kielezi).

Katika uhusika huu, kiima cha kitenzi hukubaliana kwa idadi na nafsi. Kutokana na uchambuzi wa muundo ndani wa kichwa cha habari husika, ni wazi kuwa, kitenzi taka chakubaliana na kiima wakazi katika kichwa hicho. Hivyo basi, neno wakazi ni uhusika kiima katika kichwa cha habari teule. Hiyo ndiyo sababu nomino wakazi itahama mahali ilipo katika muundo wa ndani ili kwenda kabla ya kitenzi hicho kupewa uhusika unaostahili. Fillmore (1968) anasema kuwa, uhusika kiima huhusisha vitu vyenye uhai ambavyo husisimua utendaji wa tendo. Pia, maelezo haya ni ithibati kuwa neno wakazi ndilo linalosisimua tendo la kwenda nyumbani kwa rais. Neno, wakazi lina uhai, hivyo basi ni uhusika kiima katika kichwa cha habari teule.

Uchambuzi huu hurahisisha mchakato wa kuelewa mada husika na makala yanayoihusu kwa msomaji. Kwa kuongozwa na kauli, ni nani aliyetenda tendo fulani, wasomaji mbalimbali wa magazeti huwa na fasiri sawia kinyume na wasiokuwa na kigezo cha kuwaongoza katika ufasiri wao. Hivyo basi, ujumbe sawia na faafu huwafikia watu mbalimbali kando na fasiri za kifasihi ambapo, kila msomaji huwa na uhuru wa kusoma kichwa cha makala na kukifasiri kulingana na uelewevu wake.

\section{Uhusika Mahali}

Uhusika mahali ni mojawapo wa aina sita za uhusika zilizobainika kwenye data ya utafiti. 
Kulingana na Collins (2011), uhusika mahali ni umbo la uhusika ambalo huonyesha mahali ambapo tendo lilifanyika. Vifuatavyo ni baadhi ya vichwa vya habari ambavyo muundo wake una uhusika mahali; Viongozi wa ukambani wakutana na rais ikulu, Raila aenda kuwakilisha nchi mazishini na Nia yetu si kumng'oa Uhuru madarakani Tangatanga.

Muundo wa uhusika mahali hujibu swali; tukio lilitukia wapi? Kigezo cha mahali ambapo kitendo kilitendeka huongoza mchakato mzima wa muundo wa uhusika. Kichwa kifuatacho cha habari kimechambuliwa ili kuwakilisha mchakato mzima wa muundo wa uhusika mahali; Raila aenda kuwakilisha nchi mazishini. Tukio lenyewe ni wakilisha ilhali mahali pa tukio kutendekea ni mazishini. Hivyo basi, neno mazishini ni uhusika mahali. Hali hiyo imewakilishwa na miundo ifuatayo.

\section{Kielelezo 2: Utiaji uhusika mahali}

- Muundo nje

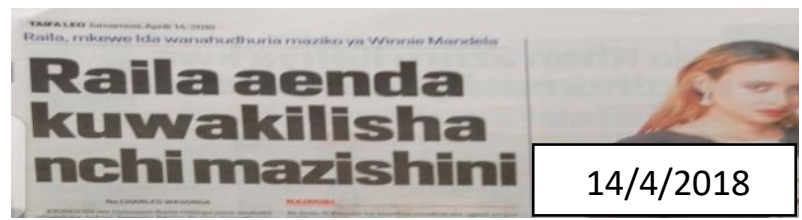

Picha hii imewakilisha muundo nje wa mojawapo ya data teule iliyokusudiwa kuchambuliwa kwa ajili ya kubainisha uhusika mahali. Kutokana na muundo huu, mtafiti ameunda muundo ndani wa kichwa cha habari teule kwa ajili ya kubainisha ujumbe ubebwao na kichwa hicho.

\section{- Muundo ndani}

Vipashio vyote vya lugha vinavyoungana na kuunda kichwa cha habari teule vimepewa maana. Neno nchi lina maana tendea ilhali kitenzi ni yawakilishwa. Kihusishi na ni ishara uhusika huku neno Raila ikiwa na maana ya uhusika kiima. Neno mazishi laashiria uhusika mahali.

Mchoroti wa muundo wa ndani wa kichwa cha habari teule ufuatao unathibitisha jinsi kanuni tatu kuu za utiaji uhusika katika sentensi hutenda kazi. Katika kanuni ya kwanza, kichwa hiki cha habari kimeundwa kwa sehemu ya hali ambayo imehusisha kigezo cha wakati uliopo na sehemu ya kauli iliyohusisha kitenzi kikuu na mfuatano wa dhana za uhusika. Kanuni ya pili imebainisha kitenzi kikuu kuwa neno wakilisha huku mfuatano wa dhana za uhusika yambwa, kiima na mahali ukikiandama kitenzi hicho. Kulingana na Fillmore (1968), uhusika unapotiwa katika sentensi, ishara uhusika hutanguliza kirai nomino kama inavyobainishwa na kanuni kwenye mchoroti wa pili.

\section{Mchoroti 2: Utiaji uhusika mahali}

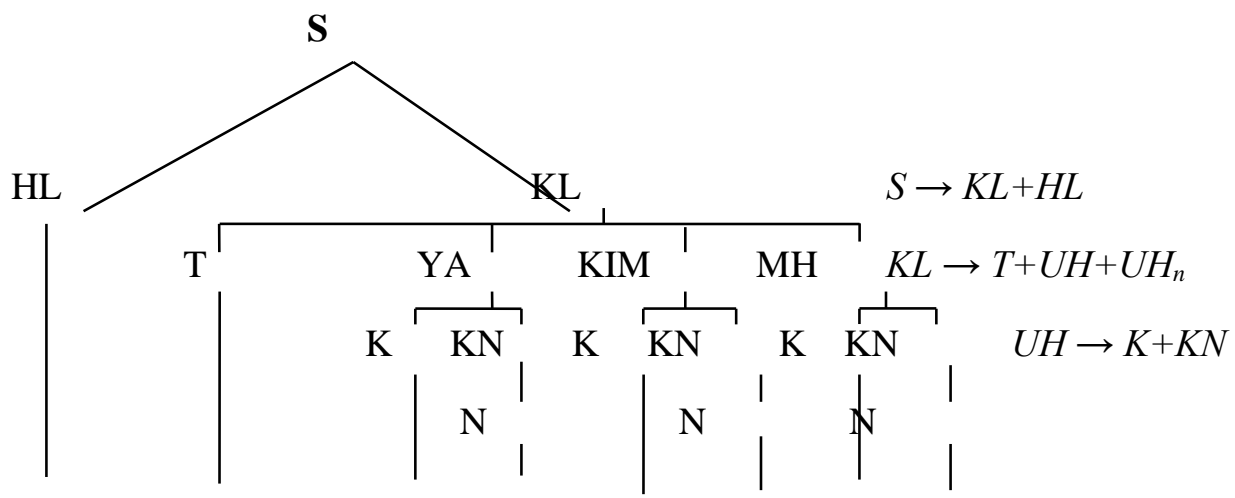

Uliopo wakilisha $\varnothing$ nchi na Raila kwa mazishi

Kigezo cha mahali ambapo kitendo kilifanyika ndicho huongoza mchakato mzima wa utiaji uhusika kwa aina hii ya uhusika. Tendo kuu husika katika kichwa cha habari teule ni wakilisha. Mtendaji amelitenda tendo hilo mazishini. Hivyo basi, mahusiano ya moja kwa moja kati ya kitenzi na nomino ya kawaida inayoashiria mahali hubainisha uwepo wa uhusika mahali katika sentensi. Mahusiano haya hurahisisha utiaji wa uhusika mahali katika sentensi. Hivyo basi, neno 
mazishi hubakia kuwa uhusika mahali katika kichwa teule cha habari.

Msomaji wa gazeti la Taifa Leo anayefahamu vyema jinsi ya utiaji uhusika katika sentensi huwa ni mwepesi wa kuelewa kinachozungumziwa na mwandishi wa makala kwa kukisoma kichwa cha habari pekee. Ufasiri wa kisarufi hujikita kwenye kigezo, mahali tendo lilitendeka. Hivyo basi, kigezo hiki huibua usawia katika ufasiri wa maana katika makala teule kwa wasomaji wote.

\section{Uhusika Yambwa}

Uhusika wa aina hii pia ulipatikana katika data iliyokusanywa. Uhusika yambwa ni aina nyingine ya uhusika aliobainisha Fillmore (1968). Huu ni uhusika ambao umbo lake la uhusika huwekwa kwenye nomino au kiwakilishi chake ili kuonyesha kwamba nomino au kiwakilishi hicho ni yambwa katika sentensi. Kwa ufupi, ni nomino au kiwakilishi kinachopokea pigo katika sentensi. Baadhi ya vichwa vya habari za siasa vilivyo na muundo wenye uhusika yambwa ni; Wapiga kura kusajiliwa leo, Ruto atetewa kuhusu 2022 na Chama cha Jubilee chateua mwanawe Too kuwa diwani.

Kwa kurejelea kichwa cha habari; Chama cha Jubilee chateua mwanawe Too kuwa diwani, muundo wa uhusika yambwa unalenga kubainisha neno mwanawe Too kama neno linalopokea pigo katika kichwa hicho.

\section{Kielelezo 3: Utiaji uhusika yambwa}

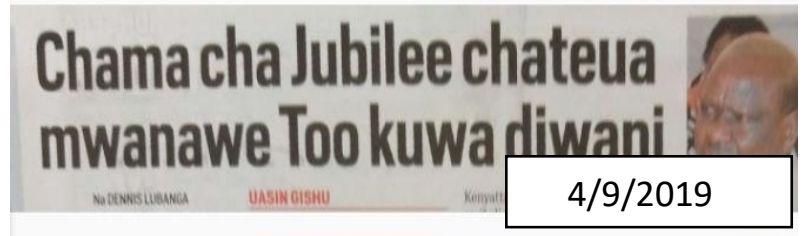

Kichwa cha habari kilichoko kwenye picha ya gazeti iliyotangulia maelezo haya huwakilisha yale maoni ya mwandishi baada ya kufanyiwa mabadiliko. Kutokana na muundo huu, mtafiti amebuni muundo ndani wake kwa ajili ya kudondoa maana ifungamanayo na kichwa hiki.

- Muundo ndani

Kila kipashio kiundacho muundo ndani wa kichwa cha habari teule kina dhima yake. Kichwa cha habari, mwanawe Too ateuliwa na chama cha Jubilee kuwa diwani kimegawanywa kwa sehemu mbili kuu: sehemu ya hali, ambayo inahusisha kigezo cha wakati uliopo na sehemu ya kauli ambayo inahusisha kitenzi kikuu na mfuatano wa dhana mbalimbali za uhusika. Kitenzi kikuu katika sehemu ya kauli ni ateuliwa ilhali aina za uhusika unaofuatana ni pamoja na; uhusika yambwa, uhusika kiima na uhusika tokeo. Ishara kapa ya uhusika imetanguliza kirai nomino mwanawe Too katika utiaji uhusika yambwa kwenye muundo huu wa uhusika kama inavyodhihirishwa. Mtafiti amezingatia utendakazi wa kanuni tatu kuu za utiaji uhusika zake Fillmore (1968) alizobainisha katika modeli yake ya mfumo wa uhusika.

- Muundo nje

Mchoroti 3: Utiaji uhusika yambwa

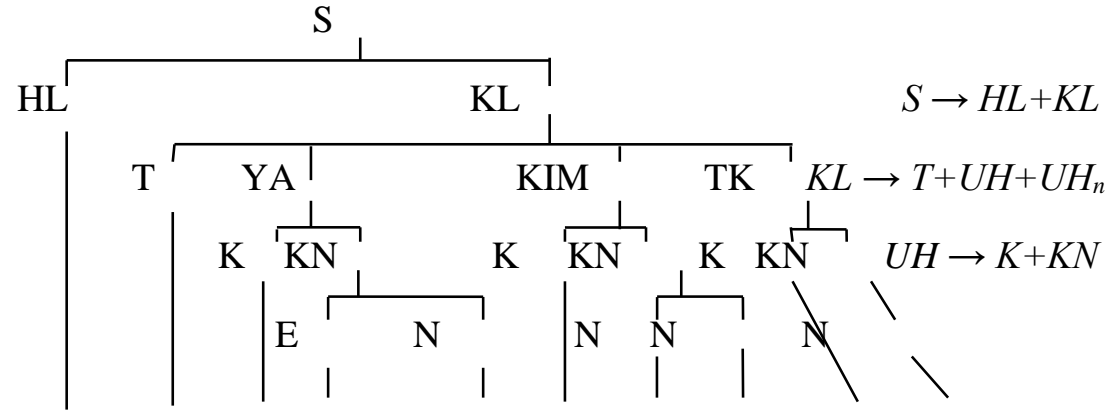

Uliopo teua $\varnothing$ mwanawe Too na chama Jubilee kuwa diwani 


\section{Tanbihi: TK (Uhusika Tokeo)}

Neno linalopokea pigo katika kichwa hiki cha habari ni Mwanawe Too. Hivyo basi, hili ndilo neno lililo na uhusika yambwa kwa mujibu wa maelezo ya Fillmore (1968) kuhusu dhana ya uhusika yambwa.

Kwa kufahamu dhana ya uhusika, wasomaji wa gazeti huongozwa na kauli, ni nani aliyepokea pigo la kitendo fulani. Kauli hii huleta usawa wa maana kisemantiki kutoka kwa msomaji mmoja hadi mwingine kwani, mahusiano kati ya kitenzi kikuu na yambwa huwa wazi. Jambo hili husababisha upitishaji wa taarifa sawia za habari kwa wananchi na kuondoa utata wa fasili mbalimbali za kichwa kimoja.

\section{Uhusika Tendea}

Hili ni umbo la uhusika ambalo huonyesha mhusika anayeathiriwa na jambo fulani. Baadhi ya vichwa teule vya habari vilivyoko katika gazeti la Taifa Leo vyenye uhusika tendea ni kama ifuatavyo; Wezi waburudika kabla kuibia mwanasiasa, UhuRuto wamefanya nifilisike, afichua Joho na Si ustaarabu wanasiasa kutimua walimu wapya.

Kwa kurejelea kichwa cha habari; Si ustaarabu wanasiasa kutimua walimu wapya, athari ya kitendo timua hujitokeza kwa maneno walimu wapya. Hivyo basi, uhusika tendea katika kichwa hiki upo kwa maneno, walimu wapya.

\section{Kielelezo 4: Utiaji uhusika tendea}

- Muundo nje

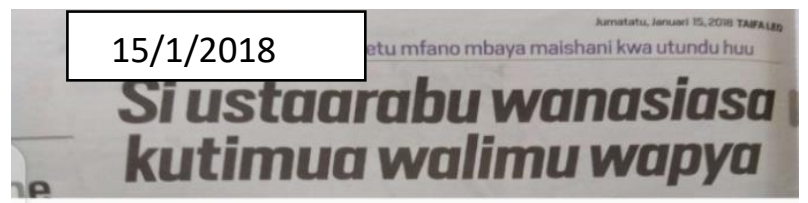

Kichwa hiki cha habari kimetumika kama kielekezo cha kuunda muundo ndani kwa ajili ya uchambuzi na utiaji uhusika.

- Muundo ndani

Uchambuzi wa muundo ndani ufuatao umeangazia jinsi uhusika tendea hutiwa katika vichwa vya habari za siasa. Utiaji uhusika tendea huu umeongozwa na kanuni tatu kuu za utiaji uhusika zilizoasisiwa na Fillmore (1968). Kanuni ya kwanza imekigawa kichwa cha habari kwa sehemu mbili kuu, ambazo ni: kauli na hali. Sehemu ya hali ina kigezo cha wakati uliopo pekee ilhali sehemu ya kauli ina kigezo cha kitenzi na mfuatano wa aina mbalimbali za uhusika. Kitenzi kikuu timua kimeandamana na kitenzi kishirikishi kipungufu $n i$, aina mbalimbali za uhusika kama vile; uhusika tokeo, uhusika tendea na uhusika kiima. Kila aina ya uhusika imetangulizwa na ishara ya uhusika ambayo huonyesha kirai nomino ambacho chafaa kutiliwa uhusika. Kwa kutolea mfano; kirai nomino, walimu wapya, ambacho kimepewa maana ya uhusika tendea kimetangulizwa na ishara kapa ya uhusika.

\section{Mchoroti 4: Utiaji uhusika tendea}

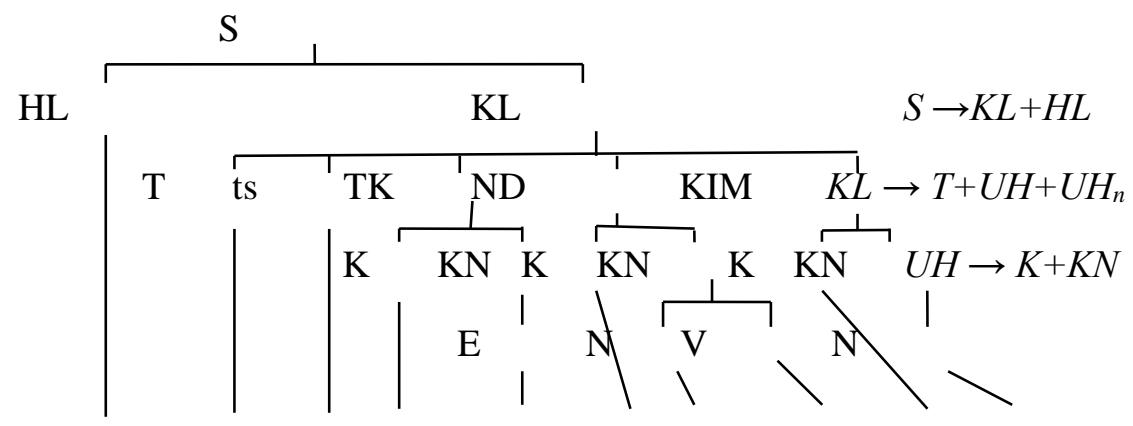

Uliopo timua ni $\varnothing$ ustaarabu $\varnothing$ walimu wapya na wanasiasa

Tanbihi: ts (Kitenzi kishirikishi kipungufu), ND (Uhusika tendea), na V (Kivumishi)
Fillmore (1968) anasema kuwa, uhusika tendea huhusisha uhusiano usiokuwa wa moja kwa moja kati ya yambwa katika sentensi na kitenzi. Kutokana 
na maelezo haya, uhusika tendea katika kichwa hiki cha habari, hutukia kwa neno walimu wapya.

Usawia wa kimaana kwa wasomi wa magazeti huongozwa na kigezo cha neno lililopokea pigo. Kisarufi, dhana ya uhusika yambwa hufafanua wazi uhusiano uliopo kati ya kitenzi na yambwa katika sentensi, hivyo basi, mahusiano haya huleta uelewevu bia kwa wasomi wote hivyo kuondoa utata wa kimaana.

\section{Uhusika Tokeo}

Mtafiti alibaini uhusika tokeo kama aina nyingine ya uhusika alioupata katika data yake. Uhusika tokeo ni umbo la uhusika ambalo hubainisha matokeo au zao la mwisho la kitendo. Vifuatavyo ni baadhi ya vichwa teule vya habari za siasa vilivyoko katika gazeti la Taifa Leo; Wapwani wabuni muungano wao, Miguna ageuzwa kipenzi cha Raila, na Tangatanga yadai wakuu walileta fujo.

Maneno yaliyopigiwa mstari katika vichwa teule vya habari huwakilisha dhana ya uhusika tokeo. Kwa kurejelea kichwa cha habari; Wapwani wabuni muungano wao, neno muungano wao ndilo zao la mwisho la kitendo buni.

\section{Kielelezo 5: Utiaji uhusika tokeo}

- Muundo nje

\section{Mchoroti 5: Utiaji uhusika tokeo}

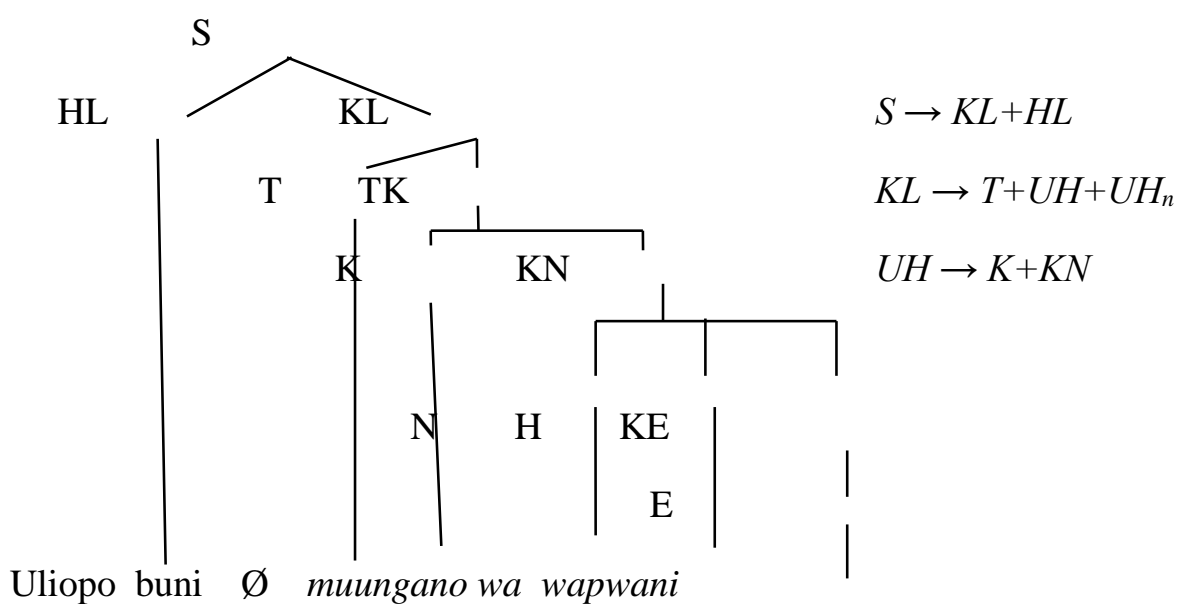

$\varnothing$ muungano wa wapwani

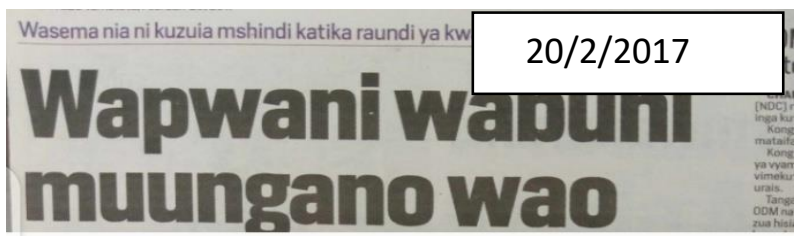

Kichwa hiki cha habari ni mojawapo ya data teule ya kubuniwa muundo ndani. Muundo nje wa kichwa hiki unaashiria yale mawazo ya tendo baada ya kuandikwa. Kisintaksia, huu ni muundo ambao umewasilisha sentensi sahihi na kamilifu kisarufi.

- Muundo ndani

Muundo ndani wa kichwa cha habari teule umeundwa kwa vipashio viwili. Kipashio cha kwanza ambacho ni muungano wa wapwani kina uhusika tokeo ilhali kipashio cha pili, wabuniwa ndicho kitenzi kikuu katika kichwa cha habari teule.

Kichwa cha habari, Muungano wa wapwani wabuniwa kimegawanywa katika sehemu mbili kuu. Sehemu ya kwanza ni hali. Hii ni sehemu iliyotawaliwa na kigezo cha wakati uliopo pekee. Sehemu ya pili ni kauli. Katika sehemu hii, kitenzi kikuu na mfuatano wa uhusika hutawala. Kitenzi kikuu, wabuniwa na uhusika tokeo, muungano wa wapwani umethibitisha utendakazi wa kanuni ya pili ya utiaji uhusika. Ishara kapa ya uhusika tokeo imetanguliza kirai nomino, muungano wa wapwani ambacho ndicho uhusika tokeo katika kichwa hiki cha habari. Mchoroti ufuatao umethibitisha jinsi uhusika tokeo huwekwa katika kichwa cha habari za siasa kwa kuzingatia tokeo la mwisho la tendo.

65 | This work is licensed under a Creative Commons Attribution 4.0 International License. 


\section{Tanbihi: KE (Kirai Kielezi)}

Zao la mwisho la uhusiano wa moja kwa moja kati ya kitenzi, buni na nomino, Wapwani, ni muungano wa wapwani. Tofauti iliyopo kati ya muungano huu na mingine ya kisiasa ni kuwa, muungano huu unamilikiwa na wapwani. Kwa hivyo, ni wazi kuwa, muungano wa wapwani ni uhusika tokeo katika kichwa cha habari teule.

Kwa kutambua tokeo la mwisho la kitenzi katika sentensi husika, maana kisemantiki ya sentensi hubainika. Tokeo la mwisho ndilo nguzo kuu katika ufasiri wa maana kisemantiki wa vichwa vya habari teule, hivyo basi, maana sawia hujitokeza wakati wa ufasiri kati ya wasomi. Usawia huu huleta uelewevu unaooana kwa kila mtu, hivyo, kupitisha ujumbe sawia wa habari na kuondoa utata wa ufasiri wa kimaana wa kichwa cha habari teule.

\section{Uhusika Ala}

Kwa mujibu wa Collins (2011), uhusika ala ni umbo la uhusika ambalo huonyesha kifaa kilichotumika katika utekelezaji wa kitendo au jambo fulani. Kifaa hicho hutumika katika kusababisha utokeaji wa tendo au hali ambayo inadokezwa na kitenzi. Ifuatayo ni mifano ya baadhi ya vichwa vya habari za siasa zenye muundo wa uhusika ala; Mwanafunzi aliuawa kwa risasi, NASA waache mizaha ya kutumia Bibilia kujiapisha na Teknolojia ya Blockchain itumiwe kuzima ufisadi. Muundo wa uhusika ala wa kichwa cha habari; Teknolojia ya Blockchain itumiwe kuzima ufisadi umetumika kama mfano ili kuonyesha jinsi uhusika huu hujitokeza katika sentensi.

\section{Kielelezo 6: Utiaji uhusika ala}

- Muundo nje

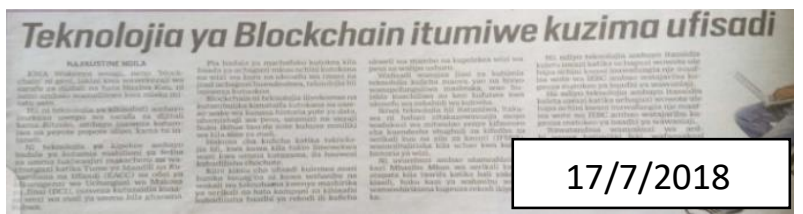

Muundo huu umetumiwa kama kielelezo cha kudondoa muundo ndani wa kichwa cha habari husika ndipo mchakato wa utiaji uhusika katika kichwa hiki ufanikishwe.

\section{- Muundo ndani}

Mchoroti wa uchambuzi wa muundo ndani ufuatao umefanikisha utendakazi wa kanuni tatu kuu za utiaji uhusika katika sentensi kwa mujibu wa Fillmore (1968). Maandishi ya mlazo yaliyo upande wa kushoto ndiyo kanuni tatu kuu za utiaji uhusika ilhali uchambuzi ulioko katika michoroti unaashiria jinsi kanuni hizo hutenda kazi. Kanuni ya kwanza imekigawa kichwa cha habari teule katika sehemu mbili kuu ambazo ni hali na kauli. Sehemu ya hali ina kigezo cha wakati uliopo pekee ilhali sehemu ya kauli ina vigezo vya kitenzi kikuu; uzimwe na mfuatano wa aina mbili za uhusika; uhusika yambwa na uhusika ala. Hata hivyo, muundo wa uhusika huu umelenga kubainisha utiaji wa uhusika ala katika mchoroti huu. Hivyo basi, kwa kuzingatia kigezo cha ala iliyotumika kutendea kitendo lengo kuu katika mchoroti huu ni kubainisha uhusika ala. Kanuni ya tatu imetumika kuonyesha utambuzi wa uhusika katika sentensi. Katika kanuni hii, ishara ya uhusika imetanguliza kirai nomino kilicho na uhusika lengwa. Kwa mfano; ishara ya uhusika kwa imetanguliza kirai nomino teknolojia ya blockchain ambacho ndicho uhusika ala katika kichwa hiki cha habari. 


\section{Mchoroti 6: Utiaji uhusika ala}

HL

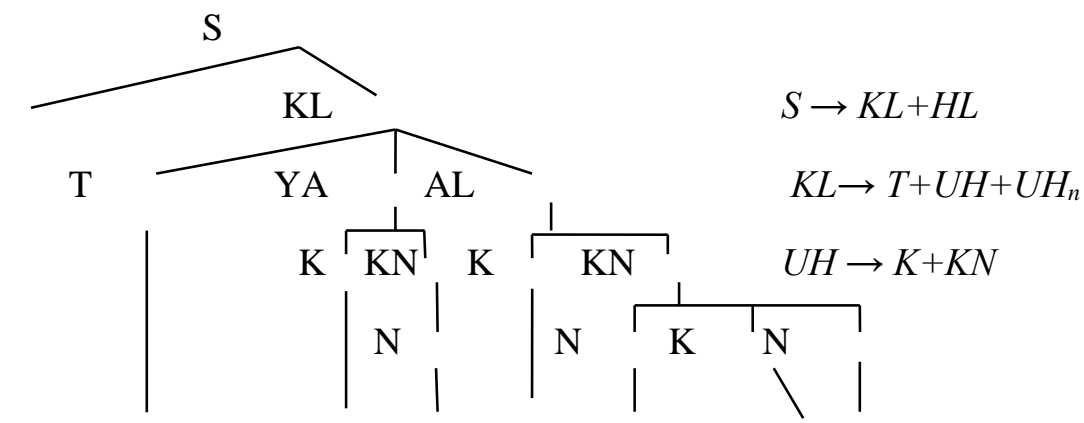

Uliopo uzimwe $\varnothing$ ufisadi kwa teknolojia ya blockchain

Tanbihi: AL (Uhusika Ala)

Tukio linalodhamiriwa kutendwa ni uzimaji. Kinachodhamiriwa kuzimwa ni tendo la ufisadi nchini Kenya. Kifaa kinachopendekezwa kitumike katika utekelezaji wa tukio hili ni teknolojia ya blockchain. Hivyo basi, teknolojia ya blockchain ni uhusika ala katika kichwa cha habari husika.

Nguzo kuu katika kubaini uhusika ala ni kujibu swali; ni kifaa gani kilichotekeleza tukio fulani? Msomaji wa gazeti anayefahamu jinsi ya kutia uhusika ala katika sentensi huwa mwepesi wa kutambua suala kuu linalokisiwa kuzungumziwa katika makala yote. Ufahamu huu hurahisisha uelewaji wake kuhusu suala linalogusiwa na kuleta fasili moja ya maana kisemantiki katika kichwa cha habari teule.

\section{Hitimisho}

Makala hii imebainisha dhana sita za uhusika kwa mujibu wa Fillmore (1968). Dhana hizo za uhusika ni pamoja na: uhusika kiima, uhusika mahali, uhusika yambwa, uhusika tendea, uhusika tokeo na uhusika ala. Pia, muundo wa ndani na wa nje wa aina hizi za uhusika umeonyeshwa kwa uwazi. Muundo nje umewakilishwa kwa picha za vichwa vya habari teule vilivyoko katika magazeti ya Taifa Leo huku muundo ndani ukiundwa kwa kuzingatia ujumbe ulioko katika picha hizo. Imebainika wazi kuwa, mahusiano kati ya kitenzi na nomino au kiwakilishi chake katika sentensi hubainisha aina ya uhusika.

Kila aina ya uhusika hutambulishwa kwa jibu la swali maalum kama ifuatavyo: jibu la swali, kitendo kilitendwa na nani huwa uhusika kiima ilhali jibu la swali kitendo kilitendewa wapi hubainisha uhusika mahali. Jibu la swali, ni nani aliyepokea pigo katika tukio huwa uhusika yambwa huku uhusika tendea ukibainishwa kwa kigezo cha mwathiriwa wa tendo. Zao la mwisho la tukio linapobainishwa katika sentensi huashiria uhusika tokeo huku kifaa kilichotumika kufanyia tendo kikidhihirisha uwepo wa uhusika ala. Kwa kuzingatia maswali haya, wasomaji wa magazeti wataweza kudondoa maana sawia ya vichwa hivyo vya habari na kupunguza utata wa kimaana baina yao. Mfumo wa modeli ya uhusika yake Fillmore (1968) na kanuni tatu kuu za utiaji uhusika, zimeongoza mchakato mzima wa utiaji uhusika kama ilivyothibitishwa katika michoroti.

\section{Marejeleo}

Chafe, W. L. (1970). Meaning and the Structure of Language. Chicago:

University of Chicago Press.

Chomsky, N. (1965). Aspects of Theory of Syntax. Cambridge: Cambridge University Press.

Collins, H. (2012). Collins English Dictionary. Wellington. New Zealand Press.

Fillmore, C. J. (1968). The Case for Case in Bach and Harms (Ed.). Universal in Linguistic Theory. New York: Holt Rinehart and Winston Press.

Massaba, D.P.B (2012). Misingi ya Fonolojia. Dar es Salaam: TATAKI.

Wamitila, K.W. (2003). Kamusi ya Fasihi. Istilahi na Nadharia. Nairobi: Focus Press. 